\title{
Die kleinen politischen Parteien Mexikos - Besprechungsaufsatz
}

\author{
von Gerhard Scheffler
}

Die Klassifizierung einer politischen Partei als klein ist hier nicht nur als Frage nach ihrer zahlenmäßigen Anhängerschaft und Innehabung von Parlamentssitzen, d. h. also lediglich quantitativ zu verstehen, sondern qualitativ ${ }^{1}$ als eine Frage der politischen Relevanz im Sinne der Möglichkeit, nicht unerheblich auf die die Nation integrierenden und repräsentierenden Vorgänge Einfluß nehmen zu können ${ }^{2}$. Angesichts der jahrzehntelangen Vorherrschaft des regierenden Partido Revolucionario Institucional - PRI - auf allen Ebenen des staatlichen, gewerkschaftlichen und bäuerlichen Lebens Mexikos und seiner erdrückenden parlamentarischen Mehrheiten im Bund und in den Einzelstaaten ${ }^{3}$ läßt alle anderen politischen Parteien gerade auch im erwähnten substantiellen Sinne als klein, d. h. wahrhaft bedeutungslos erscheinen. Dies gilt auch für den Partido Acción Nacional - PAN -, der immerhin über fast das gesamte Land in den städtischen Mittelschichten ein beachtliches, aber im Mehrheitswahlrecht nicht durchschlagendes Wählerpotential hat und von seiner zahlenmäßigen Größe als mittlere Partei zu begreifen wäre. Angesichts seiner grundsätzlichen Opposition zum PRI hat der PAN mangels Mitwirkungsmöglichkeiten weniger Einfluß als etwa die mit dem PRI zusammenarbeitenden Mini-Parteien. Die parlamentarische Parteienlandschaft läßt sich unterteilen in I. die eigenständige Oppositionspartei PAN, II. die bisher registrierten Parteien Partido Popular Socialista - PPS - und Partido Auténtico de la Revolución Mexicana - PARM - sowie III. die neuen oder erstmals registrierten Parteien.

* Zugleich eine Besprechung von

José Ángel Conchello u. a., Los partidos políticos de México, México 1975;

Ravael Avalos Marron, Los Partidos Políticos Actuales en la Constitución Mexicana, México 1977;

Daniel Moreno, Los partidos políticos del México contemporáneo, 7. Aufl. México 1979;

Manlio Fabio Murillo Soberanis, La Reforma Política Mexicana y el Sistema Pluripartidista, México 1979;

Octavio Rodríguez Araujo, La reforma política y los partidos en México, México 1979;

Volker G. Lehr, Der mexikanische Autoritarismus, München 1981.

1 Insofern stellt der Begriff "klein" auch bei sonst formaler Gleichstellung mit den entsprechenden "großen" Vertretern eine durchaus juristische Kategorie dar. Vgl. etwa die Gegenüberstellung der Großkirchen und der kleinen Religionsgesellschaften im Rahmen des Art. 140 GG, dazu Martin Heckel, Staat - Kirche - Kunst, Tübingen 1968, S. $221 \mathrm{~m}$. w. Nw.

2 Das Beispiel der F.D.P. als quantitativ kleine, jedoch kraft ihrer unentbehrlichen Mittelstellung qualitativ so einflußreichen Partei drängt sich als Anschauungsmaterial auf.

3 So stellte der PRI neben dem Staatspräsidenten alle Senatoren (in der 2. Kammer), bis $1976 \mathrm{mehr}$ als $80 \%$ aller Bundes- und Landesdeputierten und 2348 Bürgermeister gegenüber 26 von der Opposition (Stand 1974). 
Die Gründung ${ }^{4}$ des PAN ${ }^{5}$ im September $1939^{6}$ durch Manuel Gómez Morín 7 ist auf dem Hintergrund schwerer Auseinandersetzungen zwischen der antiklerikalen Revolutionsregierung und den militanten Katholiken zu sehen, die sich zum Aufstand der cristeros (Anhänger von Christus dem König) in den westlichen Bundesstaaten im Jahre 1929 steigerten ${ }^{8}$. Der Kampf endete mit Abschluß des vom US-amerikanischen Botschafter Morrow $^{9}$ vermittelten modus vivendi vom 21. Juni $1929^{10}$ zwischen der Regierung Portes Gil und dem Episkopat. Dieser modus sah u. a. die Respektierung der staatlichen Gesetze durch die Kirche einerseits und die Möglichkeit öffentlicher und gemeinsamer Kritik an politischen Entscheidungen andererseits vor. Dies war die Grundlage für die erlaubte $^{11}$ Gründung einer klerikal-bürgerlichen Partei im nicht mehr in Frage gestellten System $^{12}$, der PAN als "oposición leal ${ }^{13}$. Diese Partei konnte dank ihres eigenen politischen Vorfeldes im klerikalen ${ }^{14}$ und konservativen Bereich mit verdeckter Unterstützung der katholischen Kirche und einiger Unternehmer nennenswerte Stimmenanteile im Lande erringen ${ }^{15}$ und in den Städten ${ }^{16}$ in den Mittelschichten Anhänger gewinnen. Im

4 Vor dem Inkrafttreten des Wahlgesetzes v. 4. 12. 1951 genügte zur Anerkennung als Partei die Mitgliedschaft von 100 Bürgern, vgl. zur Wahlrechtsentwicklung Antonio Brambila, in: línea H. 20 (März/April 1976), S. 37 ff. (44).

5 Der Name Acción Nacional lehnt sich deutlich an den der französischen Organisation "Action Française" an, ohne deren reaktionäre Doktrin übernehmen zu wollen.

6 Avalos M. a.a.O., S. 107. Diese Lizenziatsarbeit beschäftigt sich (zeitlich bedingt) mit den bisherigen registrierten Parteien. - Direkter Vorgänger war der am 23. 5. 1937 in León/Guanajuato gegründete Partido Acción Nacional y Partido Revolucionario Anticomunista (PRAC), Murillo S. a.a.O., S. 57/8.

7 Gómez M., geb. 27. 2. 1897, gest. 19. 4. 1972, war 1933/4 Rektor der UNAM, Roderic Ai Camp, Mexican Political Biographies, Tucson/Arizona 1976, S. 134, politisch hervorgegangen aus der 1934 gegründeten militärischen Organisation "La Base", Rodríguez A. a.a.O., S. 126.

8 Zur Cristero-Rebellion grundlegend Jean Meyer, The Cristero Rebellion, London-New York-Melbourne 1976. Zur ideologischen Herkunft der Christero-Bewegung in den Gedanken des französischen Jesuiten-Paters Bernardo Bergöend vgl. Roberto Blanco Moheno, Cronica de la Revolución Mexicana, Bd. III: Vasconcelos, Calles, Cárdenas; Mexico 1967, S. 172 f.

9 L. Ethan Ross, in: HAHR Bd. 38 (1958), S. 506 ff. (516); Stanley Robert Ellis, in: HAHR Bd. 38 (1958), S. 482 ff. (485 ff.).

10 In Form von Statements der Staatsregierung einerseits und des Erzbischofs Ruiz andererseits, Text bei Stanley R. Ellis, in: HAHR Bd. 38 (1958), S. 503/4 mit Anm. 34 ebd.

11 Die Verwendung eines religiösen Namens zu politischen Zwecken war und ist verboten, Art. 130 Abs. XIV der Verfassung v. 5. 2. 1917, so daß eine Benennung wie "Partido Católico Nacional" nicht in Frage käme, im Anschluß an eine 1911 gegründete Partei, die 1912 in Jalisco und Zacatecas große Wahlsiege erringen konnte.

12 Dafür gingen nunmehr die Auseinandersetzungen um die Schule weiter, vorangetrieben von dem seit Okt. 1931 amtierenden Erziehungsminister Narciso Bassols, St. E. Hilton, in: The Americas Bd. XXI (1964/5), S. 175 ff. Im Dez. 1933 wurde Art. 3 der Verf. um das Gebot der sozialistischen Erziehung ergänzt, D. O. v. 13. 12. 1934, S. 849.

13 Dazu Soledad Loaeza, in: La vida política en México (1970-1973), México 1974, S. 101 ff. (108ff.).

14 Dazu José Gutiérrez Casillas, Historia de la Iglesia en México, México 1974, S. 440.

15 Der PAN errang 1946 in den Wahlen zur Deputiertenkammer, dem Bundesparlament, bei 2,37\% Stimmenanteil 4 Direktmandate; 1949: 5,2 \% und 4 Mandate; 1952: 8,7 \%; 1955: 9,17\%; 1958 bei 10,2 \% nunmehr 6 Mandate (u. a. eines in Zacatecas); 1961 bei 7,57 \% 5 Mandate, 1964 - nach Einführung des diputado del partido - errang der PAN bei 11,51 \% Stimmen 2 Direktmandate und das Maximum von 18 Ergänzungsmandaten; 1967 bei nunmehr 12,29 \% immerhin ein Direktmandat und 19 Ergänzungsmandate; 1970: 13,58 \% mit 
bisherigen Mehrheitswahlrecht war allerdings an eine direkte Umsetzung der Stimmanteile in Deputiertensitze nicht zu denken. Ebensowenig konnte der PAN bei den alles entscheidenden, alle sechs Jahre stattfindenden Wahlen zum Staatspräsidentenamt seinen Kandidaten als ernsthafte Konkurrenz aufbauen. Der 1952 erstmals vom PAN aufgestellte Kandidat Efraín González Luna erreichte 285555 Stimmen $=7,8 \%{ }^{17}$ nach Adolfo Ruiz Cortines mit 74,3\%; 1958 errang Luis H. Alvarez 9,4 \% gegenüber Adolfo López Mateos mit 90,4 \%. 1964 war es nicht viel anders: José González Torres ${ }^{18}$ erreichte nur 10,9\% der Stimmen gegenüber Díaz Ordaz mit 88,9\%. 1970 erreichte Efraín González Morfín mit 2 Mio. Stimmen gegenüber Luis Echeverría Alvarez $(84,6 \%)$ immerhin 13,8 \% Anteil ${ }^{19} .1976$ indes konnte der PAN aufgrund interner Streitigkeiten ${ }^{20}$ keinen Kandidaten aufstellen ${ }^{21}$. Wichtiger für den PAN als Oppositionspartei, zugleich jedoch auch kennzeichnend für das politische System ist die Situation in den Bundesstaaten. Hier gelang es dem PAN schwerpunktmäßig mit populären Kandidaten, den PRI in Bedrängnis zu bringen ${ }^{22}$; zu einem Regierungswechsel ist es gleichwohl nicht gekommen $^{23}$. Der PAN mußte um seiner eigenen Existenz willen die Unantastbarkeit des Regimes von gewissen Positionen an aufwärts anerkennen und konnte dafür ungefährdet wenigstens die aus eigener Kraft erworbenen anderweitigen Positionen, wie etwa Bürgermeisterämter und Deputiertensitze, einnehmen ${ }^{24}$. In den Deputiertenwahlen vom 1. Juli 1979 indes verlor der PAN in seinen bisherigen Hochburgen im Distrito Federal (von $21,27 \%$ im Jahre 1976 auf 17,23\%) und Puebla (von 12,65\% auf 11,65\%) für jedoch in den nördlichen Bundesstaaten hinzugewinnen (so in Baja California Norte von $15,6 \%$ auf $18,76 \%$, in Sonora erreichte der PAN $16 \%{ }^{26}$. Der PAN dürfte hier allein von den Protestwählern profitiert haben, z. T. ohne Konkurrenz von links (Kommunisten) und rechts wie im Zentrum des Landes, so die Erklärung von Segovia ${ }^{27}$.

20 Ergänzungsmandaten, vgl. Rodríguez A. a.a.O., S. 126 ff., der jeweils in einem historischen Teil zu jeder der von ihm behandelten Parteien dieses statistische Material aufbereitet.

16 Rafael Segovia, in: La vida política en México (1970-1973), Mex. 1974, S. 49 ff. (65).

17 Diese Stimmenanteile sind nicht identisch mit den dazu verschiedenen Wahlergebnissen zu den gleichzeitig stattfindenden Deputiertenwahlen, vgl. Anm. 15.

18 Gonzáles T. war der damalige militant katholische, antikommunistische Führer des PAN mit der Parole "cristianismo si, comunismo no".

19 Rodríguez A. a.a.O., S. 129.

20 Carlos Arriola, in: Foro Internacional Bd. XVII Nr. 4 (April-Juni 1977), S. 542 ff.; Kenneth F. Johnson, Mexican Democracy: A Critical View, 2. Aufl. New York-London-Sydney-Toronto 1978, S. 145 ff., Rodríguez A. a.a.O., S. $132 \mathrm{f}$.

21 Erstmals mußte der PRI-Präsidentschaftskandidat - J. López Portillo - ohne jeden Gegenkandidaten antreten, eine Schlappe auch für das System selbst, NZZ Nr. 154 v. 6. 7. 1976. Der PAN erreichte in der gleichzeitig stattfindenden Deputiertenwahl nur 8,5\% der Stimmen, das schlechteste Ergebnis seit Jahren.

22 So 1959 und 1968 in den Gouverneurswahlen von Baja California Norte, 1969 in den Wahlen von Yucatán, Miguel Ángel Granados Chapa, in: Foro International Bd. XVI Nr. 4 (April-Juni 1976) S. 429 ff. (S. $431 \mathrm{ff}$.).

23 Zu den Vorgängen 1967 in Sonora Johnson a.a.O., S. 179 f.; Granados Chapa a.a.O., S. 432.

$24 \mathrm{Da} ß$ damit dem Regime die Fassade eines funktionierenden parlamentarischen Systems gegeben wurde, ist eine im PAN heftig umstrittene Frage, Johnson a.a.O., S. 145.

25 Rafael Segovia, in: Foro Internacional Bd. XX Nr. 3 (Jan.-März 1980), S. 397 ff. (S. 401/2).

26 Segovia (wie Anm. 25), S. 402.

27 Wie Anm. 25, S. 402/3. 
Die Chancen des PAN für die Wahlen 1982 werden auch unter dem 1978 gewählten Vorsitzenden Abel Vicencio Tovar wiederum nur als grau bezeichnet werden können ${ }^{28}$.

II.

Die bislang neben dem PAN registrierten Parteien PPS und PARM sind reine Satellitenparteien des PRI mit der Aufgabe, am linken - PPS - und rechten Rande - PARM der Wahlbevölkerung oppositionelle Kräfte zu binden.

Am 20. Juni 1948 wurde als Linkspartei der Partido Popular - PP - von Vincente Lombardo Toledano ${ }^{29}$ gegründet $^{30}$, der sich auf die im Jahre 1947 vorangetriebenen Zusammenschlüsse der Liga de Acción Política unter Narciso Bassols ${ }^{31}$ mit Angehörigen der Universidad Obrera, früheren Mitgliedern des Partido Comunista Mexicano - PCM -, Studenten und Marxisten verschiedenster Prägung sowie Komitees in 27 Bundesstaaten $^{32}$ stützen konnte ${ }^{33}$. Der 1947 vorgeschlagene Programmentwurf verstand die Partei als solche der kleinen Leute ${ }^{34}$, die sich $u$. a. einer effektiven Industrialisierung verschreiben wollte. Den bei Linksparteien in Mexiko üblichen Absplitterungen ${ }^{35}$ konnte der Partido Popular dank des Zulaufs von Gewerkschaftsmitgliedern und mit dem konservativen Kurs des Staatspräsidenten Alemán V. (1946-1952) unzufriedenen Anhängern der mittlerweile in PRI unbenannten Regierungspartei überstehen ${ }^{36}$. Die Idee einer machtvollen Unterstützung durch eine neue, von der CTM unabhängigen Gewerkschaftszentrale scheiterte. Die von Lombardo Toledano gegründete Unión General de Obreros y Campesinos de México sank unter dem Druck der CTM zur Bedeutungslosigkeit ab. Der PP wurde immer mehr eine Partei der Intellektuellen, sie bekannte sich 1955 zum

28 Rafael Segovia, in: Excelsior v. 20. 2. 1981. Zu den Bewerbern um die Präsidentschaftskandidatur im PAN vgl. Excelsior v. 14. 5. 1981. S. 4 A.

29 Lombardo T. war der marxistische Führer der sich als Volksfrontbündnis verstehenden, 1936 gegründeten neuen Gewerkschaftszentrale Confederación de Trabajadores de México - CTM -, die mit der Unterstützung des progressiven Staatspräsidenten Cárdenas (1934-1940) an Macht vor den anderen Gewerkschaftsbünden gewann, Michael Domitra, Die Rolle der Gewerkschaften im mexikanischen Herrschaftssystem, Bonn-Bad Godesberg 1975, S. 83 ff.; Joe C. Ashby, Organized Labor and the Mexican Revolution under Lázaro Cárdenas, Chapel Hill 1963 (Nachdruck 1967), S. 72 ff.

30 Voran ging eine Zusammenkunft marxistischer Führer in México Ciudad v. 13.-22. 1. 1947, Avalos M. a.a.O., S. 172; Rodríguez A. a.a.O., S. 142. Am 12. 7.1948 wurde der P P registriert, D. O. v. 8. 9. 1948.

31 Dem vormaligen kirchenf eindlichen Erziehungsminister, vgl. Anm. 12, der dem Versöhnungskurs der Regierungsparteien gegenüber der Kirche nicht folgen mochte.

32 Rodríguez A. a.a.O., S. 143.

33 Moreno a.a.O., S. 248 ff.; Murillo S. a.a.O., S. $79 \mathrm{ff}$.

34 Moreno a.a.O., S. 249. Die Arbeit von M. ist historisch aufgebaut und gibt im wesentlichen Entwicklungsetappen wieder. Eine für den deutschen Leser ungewöhnliche Konstruktion, offenbar jedoch erfolgreich, da die Arbeit bereits in 7. Aufl. erscheint.

35 So traten u. a. 1954 Bassols aus und 1960 Vicente Fuentes Diaz, der später zum Generalsekretär des PRI aufstieg.

36 Für die Präsidentschaftswahlen 1952 einigten sich die Oppositionsparteien nicht auf einen gemeinsamen Kandidaten, so daß der PP seinen Präsidenten Lombardo T. aufstellte (mit Unterstützung der Kommunisten) und $1,98 \%$ der abgegebenen Stimmen erhielt. Die Partei selbst erhielt für die Deputiertenkammer überhaupt nur 0,88 \% der Stimmen, Rodríguez A. a.a.O., S. 145. 
Sozialismus und zur Philosophie des dialektischen Materialismus. Am 16. Oktober 1960 benannte sie sich in Partido Popular Socialista um ${ }^{37} .1963$ nahm sie eine weitere Splittergruppe auf, den Partido Obrero-Campesino Mexicano. Der PPS gibt sich als rein marxistische Partei (Unvermeidlichkeit der Revolution, Klassenkampf, proletarischer Internationalismus, die Partei als Avantgarde auf dem Weg zum Sozialismus) ${ }^{38}$. Im mexikanischen Mehrheitswahlrecht ${ }^{39}$ konnte der PPS bundesweit keine Bedeutung erlangen ${ }^{40}$. Durch Wahlrechtsänderung wurde auf Bundesebene der diputado de partido eingeführt: 1961 hatte neben dem PAN mit 5 Mandaten (von insgesamt 177) der PPS nur einen Abgeordneten (aus Nayarit) in die Deputiertenkammer entsenden können. Um den kleinen Parteien gleichwohl eine die erdrückende Dominanz des PRI kaschierende Repräsen$\operatorname{tanz}$ im Bundesparlament zu gewähren, wurden zusätzliche, nämlich wenigstens 5 Parteienmandate auf diejenigen Parteien verteilt, die bundesweit 2,5\% der Wählerstimmen erreichten, außerdem je 0,5 weiteren Stimmanteil einen Abgeordneten bis maximal 20, abzüglich der gewonnenen Direktmandate. Von dieser Wahlrechtsreform ${ }^{41}$ profitierte 1964 erstmals der PAN, der neben den 2 Direktmandaten 18 Parteimandate erreichte. Aber auch der PPS errang neben dem Direktmandat in Sinaloa 9 Parteimandate und der PARM 5 Parteimandate, und das, obwohl beide Parteien unter der 2,5\%-Marke mit $1,37 \%$ bzw. 0,71 \% verblieben ${ }^{42}$. Diesen Manipulationen zugunsten der beiden Satellitenparteien wird im einzelnen in der Arbeit von Lehr (S. 199 ff.) nachgegangen, deren Untertitel: "Parteien, Wahlen, Herrschaftssicherung und Krisenpotential" - wieder einmal - erst den eigentlichen Inhalt der Untersuchung wiedergibt. Diese indes wertet unter intensiven Recherchen vor Ort in Mexiko alle verfügbaren Quellen, z. B. unveröffentlichte Statistiken des Registro Nacional de Electores, über die parlamentarische Repräsentanz der politischen Parteien bis zu den Bundesdeputiertenwahlen von 1979 aus. Die Oppositionsabgeordneten werden hier erstmals vollständig namentlich (seit 1940) mit Wahlkreis und mit absoluten sowie relativen Stimmanteilen aufgeführt. Dieser Anhang (S. 306-355) allein wird künftig unentbehrliches Rüstzeug für alle Arbeiten über die politischen Parteien Mexikos sein. Dies gilt auch für die Erhebungen in den einzelnen Bundesstaaten anhand der Wahlgesetze zum Zeitpunkt $1976^{43}$ (S. 215 ff.)

37 Avalos M. a.a.O., S. 173.

38 Moreno a.a.O., S. 253. Die Partei schien eine Alternative der Linken zu sein, Arnaldo Córdova, in: Cuardernos Politicos Nr. 19 (Jan.-März 1979), S. 14 ff. (S. 32), ein Traum, der spätestens mit der Studentenbewegung von 1968 zerstob. Zur Entwicklung der politischen Studentenbewegung Luis Molina Piñeiro, Estructura del Poder y Reglas del Juego Político en México, México 1976, S. 39 ff.

39 Nach welchem zuletzt 1976 auf Bundesebene gewählt worden war in 196 Wahlkreisen. Hinzu kamen die Ergänzungs- bzw. Parteideputierten, s. o. im Text.

40 Die absoluten Zahlen sanken kontinuierlich ab, so daß angesichts der wachsenden Bevölkerung der prozentuale Stimmenanteil noch deutlicher absank. Erhielt der PPS 1973 noch 541833 Stimmen, so 1976 nur 479426 und 1979 ganze 387 036, Segovia (Anm. 25), S. 405.

41 Gesetz v. 22. 6. 1963. Dazu Eduardo Ruiz Moncayo, La Representación de Minorias an la Camara de Diputados del Congreso de la Unión, México 1971, S. $141 \mathrm{ff}$.

421967 erlangte der PPS 2,16 \%, 1970 nur 1,35 \%, 1973 wieder 3,61 \% und 1976 2,98\%. Vgl. Comercio Exterior 1979, S. 733 ff. (735).

43 Zum neueren Stand 1979/80 vgl. Gaceta informativa (Legislación y jurisprudencia) Nr. 29 (Jan.-April 1980), S. $68 \mathrm{ff}$. 
Die Herabsetzung des Quorums von 2,5\% auf 1,5\% für die Parteimandate ${ }^{44}$ - der PARM hatte 1970 nur 0,8\% erreicht - erleichterte ein besseres Abschneiden auch des PPS, der 1973 3,61 \% der Stimmen erlangte, 1976 - nach den zu erwähnenden Vorkommnissen in Nayarit - indessen bundesweit nur 2,98\%. Gelegentlich hatte der PPS regional größere Erfolge zu verzeichnen. So errang er 1975 im Bundesstaat Nayarit bei den Gouverneurswahlen die Mehrheit der Stimmen ${ }^{45}{ }^{46}$. Der Kandidat des PRI wurde jedoch zum Wahlsieger erklärt. Der PPS unter seinem Generalsekretär Jorge Cruickshank Garcia (seit 1968 nach dem Tode von Lombardo Toledano) nahm das hin ${ }^{47}$. Zum Dank ging der PRI bei den Bundesdeputierten- und Senatorenwahlen 1976 mit dem PPS eine "Koalition" ein dergestalt, daß für die 2. Kammer, die Senatorenkammer, der eine von beiden Senatorensitzen aus Oaxaca an Cruickshank fiel ${ }^{48}$. Mitglieder, die diesen Handel nicht akzeptieren mochten, verließen 1976 noch vor den Bundesdeputiertenwahlen den PPS und gründeten 1977 den Partido del Pueblo Mexicano - PPM - unter Stephens Garcia.

Die Alibifunktion der weiteren bislang registrierten Partei ${ }^{49}$, des am 28. Februar 1954 gegründeten PARM, ist auf den ersten Blick nicht derart offensichtlich. Veteranen der Revolutionszeit 1910-1916 hatten sich um den General Jacinto B. Treviño $0^{50}$ und Juan Barragán ${ }^{51}$ geschart $^{52}$, um den Revolutionsgeist aufrechtzuerhalten und die revolutionären Prinzipien der Verfassung in die Wirklichkeit umzusetzen. Was das konkret eigentlich bedeutet, ist auch und gerade aus dem Programm nicht zu ersehen ${ }^{53}$ 54: Danach wer-

44 Im Jahre 1973. Dazu Fernando J. Rivera Arteaga, Análisis Crítico del Sistema Electoral Mexicano. Las reformas políticas de 1970-1976, México 1976, S. $174 \mathrm{ff}$.

45 Granados Chapa (Anm. 22), S. 441: Nach dem of fiziellen Ergebnis entfielen auf den Gouverneurskandidaten des PRI 69762 und auf den des PPS nur 44152 Stimmen. Für die Wahlen 1981 in Nayarit forderte die Linke daher öffentliche Auszählung, Excelsior v. 20. 5. 1981, S. 18 A. Der PRI gewann wiederum.

461973 hatte er in Baja California Norte nur deshalb mit 42213 Stimmen 13,82\% Anteil erreicht, weil der PAN dort nicht kandidierte, Segovia (Anm. 25), S. 405. Ähnlich war es in Oaxaca, als der PPS 1976 nur deshalb $6,11 \%$ erreichte, weil der PAN nur in 3 von 9 Wahldistrikten kandidierte und der PRI dank der Koalitionsabsprache den PPS massiv unterstützte.

47 Was eigentlich gewesen wäre, wenn der PPS diese Manipulation nicht hingenommen hätte, wird in den politologischen Untersuchungen offengelassen. Daß der PRI jemals einen Gouverneursposten freiwillig räumen würde, wird indes allgemein verneint. Zu den internen Diskussionen im PRI Lehr a.a.O., S. 161.

48 Darüberhinaus erhielt der PPS 4 Parteimandate mehr als ihm nach dem prozentualen Stimmenanteil a n sich zustanden.

49 Am 15. 7. 1957, Avalos M. a.a.O., S. 211.

50 Geb. 11.9. 1883, gest. 6.11. 1971, Anhänger zunächst von Carranza, später von Obregón, Camp a.a.O., S. 321.

51 Geb. 30. 8. 1894, gest. 28. 9. 1974, Anhänger von Carranza, 1964-1967 und 1970-1973 Bundesdeputierter für den PARM, dessen Präsident 1957-1974, Camp a.a.O., S. 27.

52 Johnson a.a.O., S. 150. Die Gruppe um Treviño nannte sich "Hombres de la Revolución", Avalos M. a.a.O., S. 211.

53 Vgl. die Wiedergabe bei Moreno a.a.O., S. 341-344. S o tritt der PARM für die strickte Einhaltung des revolutionären Charakters der Erziehung gem. Art. 3 der Verfassung ein, Avalos M. a.a.O., S. 214. Da jedoch Art. 3 in den Auseinandersetzungen mit den militanten Katholiken in den zwanziger und dreißiger Jahren eine zentrale Rolle gespielt hat mit zahlreichen Änderungen, dazu Peter C. Stanchina, Das Verhältnis von Staat und Kirche in Mexiko, München 1978, S. 97, 121 ff., insbesondere der zeitweisen Anordnung einer "sozialistischen Erziehung", wäre es erf reulich gewesen, wenn das Programm genau sagte, welche ideologische Richtung nun der PARM verfolgt, was denn nun der revolutionäre Charakter der Erziehung ist. 
den alle grundlegenden Prinzipien der Verfassung anerkannt und verteidigt. Immerhin läßt sich etwa aus dem Bekenntnis zum Privateigentum - Art. VI Declaración de Principios - programmatisch die im parteipolitischen Spektrum als rechts vom PRI angesiedelte Stellung der Partei absichern. Unter dem erweiterten Wahlsystem des diputado de partido konnte auch der PARM einige Sitze erlangen ${ }^{55}$. Die dafür notwendige Stimmenzahl zog die Partei in regionalen Schwerpunkten ${ }^{56}$ durch ein publizitätsträchtiges Agieren auf sich. Mit spektakulären Blockaden von Wahllokalen, tätlichen Angriffen auf PRI-Anhänger ${ }^{57}$ gab und gibt sie der Regierungspartei immer wieder die Gelegenheit, ihrerseits auf Wahlbehinderungen durch den PARM hinzuweisen ${ }^{58}$, um auf diese Weise die ihr angelasteten Wahlmanipulationen ${ }^{59}$ zu relativieren und als Ausschnitt allgemeiner, nämlich auch bei anderen Parteien vorkommender bedauerlicher Entgleisungen darzustellen. Diese Auseinandersetzungen sind um so heftiger je bedeutungsloser die politische Ebene ist: Bei Gemeindewahlen gibt es häufig derartige Szenen, indes wird ein Ausufern der Tätlichkeiten alsbald und gegebenenfalls mit Militär verhindert ${ }^{60}$, auf Bundesebene schließlich unterstützt der finanziell vom PRI ausgehaltene PARM nicht anders als der PPS den Präsidentschaftskandidaten der Regierungspartei.

1977 mußte die Führungsspitze des PARM unmittelbar vom Innenministerium neu geordnet werden, indem der knapp 80jährige pensionierte General Antonio Gómez Velasco zum Vorsitzenden abgestellt wurde ${ }^{61}$. Die fortdauernden internen Auseinandersetzungen verhindern ebenfalls einen nennenswerten Einfluß im politischen Geschehen Mexikos. 1979 wurde als Interimspräsident Admiral Antonio Vázquez del Mercado gewählt ${ }^{62}$. Gleichzeitig wurde der Generalsekretär Prof. Rubén Rodríguez Lozano be-

54 Segovia (Anm. 25), S. 407, weiß den PARM auch nur als PRI ohne PRI zu definieren, d. h. ohne dessen Doktrin und Disziplin.

55 Im reinen Mehrheitswahlsystem erlangte er 1958 einen Abgeordneten in Morelos, 1964 erreichte er mit $0,71 \% 5$ Parteimandate, 1967 mit $1,4 \%$ wiederum 5 Parteimandate. 1970 mit $0,8 \%$ ebenfalls 5 Parteimandate, 1973 neben einem Direktmandat im regionalen Schwerpunkt Tamaulipas noch 6 Parteimandate (bei $1,82 \%$ ) und 1976 neben dem Direktmandat in Tamaulipas bei 2,51 \% - der höchsten Stimmenzahl im Bund, die der PARM je erreichte - 9 Parteimandate.

56 Tamaulipas und Veracruz sind die Hochburgen des PARM, zumal in Tamaulipas der PAN nicht existiert. In Tamaulipas erreichte der PARM $1973=6,41 \%, 1976=12,4 \%$ und $1979=16,84 \%$ (im Wahldistrikt I dort sogar 42,65\%), in Veracruz $1973=1,44 \%, 1976=2,43 \%$ und $1979=4,7 \%$, Segovia (Anm. 25), S. 406/7.

57 Für Nuevo Laredo in Tamaulipas vgl. Novedades v. 9. 8. 1976, S. 8.

58 So legte der PRI bei den Gemeindewahlen in Tapachula/Chiapas formellen Protest ein wegen Wahlbehinderungen durch den PARM, nachdem dieser bemängelt hatte, daß viele seiner Anhänger nicht in den Wahllisten registriert worden seien, Excelsior v. 3. 3. 1980, S. 26 A.

59 Für Tamaulipas und Oaxaca stellte der PARM 1978 eine Dokumentation der Wahlbehinderungen auf, Novedades v. 4. 1. 1978, S. 1 u. 7. Bei den Gemeindewahlen im Bundesstaat Veracruz im Okt. 1979 sah sich der PPS behindert, Novedades v. 7. 10. 1979, S. 14, ebenso wie der PARM, der dies im Kongreß von Veracruz rügte, Novedades v. 17. 10. 1979, S. 7. Für frühere Wahlmanipulationen (1973) vgl. Manfred Mols, in: MolsTobler, Mexiko. Die institutionalisierte Revolution, Köln-Wien 1976, S. 171 ff.; vor allem auch Lehr a.a.O., S. $185 \mathrm{ff}$.

60 So in Tamaulipas im Anschluß an Gemeindewahlen als dem Gouverneur auch vom PARM vorgeworfen wurde, sich am Rauschgif thandel bereichert zu haben, Latin America Political Report v. 20. 1. 1978, Bd. XII, S. $20 / 1$.

61 Zu den Vorgängen i m desolaten Führungszirkel des PARM nach dem Tode seines früheren langjährigen Vorsitzenden Barragán 1977 vgl. Lehr a.a.O., S. $156 \mathrm{f}$.

62 Novedades v. 29. 9. 1979, S. 15. 
schuldigt, 1 Mio. Pesos (d. h. 75000 DM) an Parteigeldern unterschlagen zu haben ${ }^{63}$ und Gómez V. für die Wahlmißerfolge verantwortlich gemacht.

III.

Mit der Verfassungsreform vom 6. Dezember 1977 und der Wahlgesetzänderung $1977^{64}$ traten nunmehr weitere und vor allem auch neue politische Parteien zu den Wahlen auf. Die kleineren Parteien erhielten in dem nunmehrigen kombinierten Mehrheits- und Verhältniswahlrecht die Chance, stärker in den Parlamenten ${ }^{65}$ vertreten zu sein: Auf Bundesebene werden die nunmehr regelmäßig 400 Deputierten in 300 Wahlkreisen mit relativer Mehrheit gewählt und der Rest von bis zu 100 Sitzen nach Landeslisten im Verhältniswahlrecht ${ }^{66}$. Berücksichtigt werden im Verhältniswahlrecht indes nur die Parteien, die nicht mehr als 60 Direktmandate erhalten und mehr als 1,5\% der Gesamtstimmenzahl erreichen ${ }^{67}$. Damit ist anscheinend nur der PRI von der Vergabe von Sitzen nach dem Verhältniswahlrecht ausgeschlossen, tatsächlich ist durch Art 54 Ziff. IV der Verfassung eine Begrenzung auch für etwa zu stark werdende Oppositionsparteien eingebaut: Erreichen zwei oder mehr der Minderheitenparteien zusammen 90 Direktmandate werden statt 100 nur noch 50 Sitze im Verhältniswahlrecht vergeben ${ }^{68}$.

Ein Blick auf die neue Parteienlandschaft zeigt auf der linken Seite ein besonders buntscheckiges Bild.

Althergebrachte Linkspartei ist der moskautreue Partido Comunista Mexicano PCM -. Gegründet auf der Zusammenkunft vom 15. September 191969, initiiert von einer Federación comunista del Proletariado Mexicano aus dem Distrito Federal, Delegierten des Buró Latinoamericano sowie Angehörigen der neu entstandenen Gewerkschaftszirkel, sah sich die Partei alsbald von den siegreichen Revolutionskräften verfolgt: Der erste Generalsekretär, José Allen, wurde noch 1919 als unerwünschter Ausländer von der Regierung Carránza ausgewiesen. Darüberhinaus litt die Schlagkraft der Partei unter internen Auseinandersetzungen, die of t mit dem Ausschluß von mehr Mitgliedern als in der Partei verblieben endeten. Zur Zeit des Präsidenten Cárdenas

63 Wie vorige Anm.

64 Ley Federal de Organizaciones Políticas y Procesos Electorales, D. O. v. 30. 12. 1977. Zum Inhalt Irma Fuentes, in: Novedades v. 8. 12. 1977, S. 1 und 10 f. Friedrich Wehner, Grundlagen einer mexikanischen Verfassungsgeschichte, Hamburg 1978, S. 193 ff.; Volker G. Lehr, Mexikos "reforma política ": Zur Erneuerung des mexikanischen Parteien- und Wahlsystems, in: Hanns-Albert Steger - Jürgen Schneider (Hrsg.), Wirtschaft und gesellschaf tliches Bewußtsein in Mexiko seit der Kolonialzeit, München 1980, S. 451 ff. (465 ff.).

65 Von Bundes wegen ist nach dem ergänzten Art. 115 Ziff. III Verf. dieses Systems auch in den Einzelstaaten und in Gemeinden von mehr als 300000 Einwohnern einzuführen. Als erster hat der Bundesstaat Mexico dieses Gebot im März/April 1978 erfüllt, dazu Jorge Madrazo, in: Gaceta informativa (Legislación y jurisprudencia) Nr. 31 (Sept.-Dez. 1980), S. 725 ff.

66 Art. 52 Verf.

67 Art. 54 Ziff. II Verf.

$68 \mathrm{Zu}$ den sich daraus für den PRI ergebenden Möglichkeiten vgl. Lehr a.a.O., S. 227.

69 Moreno a.a.O., S. 196; Arnaldo Martínez Verdugo gibt im Sammelband "Los partidos políticos de México" als Gründungsdatum den 24. 11 . 1919 an, a.a.O., S. 147. 
(1934-1940) - der unter Volksfrontgesichtspunkten von dem PCM unterstützt wurde ${ }^{70}$ erreichte die Partei die höchste Mitgliederzahl mit $32000^{71}$. Insbesondere von den parteiinternen Auseinandersetzungen während der Präsidentschaft von Alemán V. (1946-1952) konnte sich die Partei lange nicht erholen. Ihren einflußlosen Charakter als intellektuellen Politzirkel mußte sie sich eingestehen, wenn sie den fehlenden Kontakt zu den "werktätigen Massen" beklagte ${ }^{72}$. Einen gewissen Zulauf hatte der PCM allenfalls unter den Studenten. Die Partei weigerte sich, sich für die Wahlen nach den bisherigen Wahlgesetzen registrieren zu lassen ${ }^{73}$ und brauchte auf diese Weise ihre behauptete Stärke nicht unter Beweis zu stellen ${ }^{74}$. Sie sah sich allerdings dadurch dem Vorwurf des die Revolution verwaltenden PRI ausgesetzt, unglaubwürdig zu sein. Angesichts der ernst zu nehmenden Konkurrenz der trotzkistischen Parteien nahm nunmehr der PCM an den Wahlen 1979 teil, nachdem er durch ein Wahlbündnis mit einigen Splittergruppen seine Basis im Wahlvolk hat verbreitern können:

Der PPM - Partido del Pueblo Mexicano ${ }^{75}$ - und der PSR - Partido Socialista Revolucionario - bildeten mit dem PCM Ende Juni 1979 eine "Vereinigte Front "16 unter Einschluß des Movimiento de Acción Unidad Socialista - MAUS - ${ }^{77}$. Der PSR setzte sich bei seiner Registrierung als eine asociación für eine allgemeine Amnestie und die Stärkung der Gemeindefinanzen ein. Das disziplinierte Auftreten des gut organisierten PCM konzentriert auf die Städte mit Universitäten ${ }^{78}$ und der durch das erwähnte Wahlbündnis belegte Anspruch, eine "Koalition der Linken" zu sein, die Wahlenthaltung des PMT und die Spaltung der herkömmlichen Linkspartei, des PPS ${ }^{79}$, führte zu einem beachtlichen Wahlerfolg: 5,09\% der Zweitstimmen. Immerhin kann in der Deputiertenkammer

70 Arturo Anguiano, El Estado y la política obrera del cardenismo, 4. Aufl. México 1980, S. $106 \mathrm{ff}$.

71 Moreno a.a.O., S. 197.

72 Resolution des PCM im D. F. von 1957, abgedruckt bei Moreno a.a.O., S. $202 \mathrm{ff}$.

73 Zur offiziellen Begründung mit den administrativen Restriktionen seit 1946 zuletzt Arturo Martínez Nateras, in: El Partido Comunista Mexicano en la campaña electoral, México 1977, S. $123 \mathrm{ff}$.

74 So benannte sie 1976 als ihren Präsidentschaftskandidaten den Eisenbahnerführer Valentín Campa Salazar, ohne an den Wahlen teilzunehmen, Lorenzo Meyer, in: Comercio Exterior 1976, S. 644 ff. (645).

75 Diese 1977 durch Abspaltung vom PPS entstandene Partei hat ihren Schwerpunkt in Nayarit, wo sie trotz einhunderttausend Anhängern nicht registriert wurde, weil nicht bundesweit vertreten, Lehr a.a.O., S. 219. Demgegenüber wurde sie im Bundesstaat Baja California Sur als Landespartei am 4. 7. 1980 registriert, Gaceta informativa (Legislación y jurisprudencia) Nr. 31 (Sept.-Dez. 1980), S. 670.

76 Entwicklungspolitik kommunistischer Länder II/1978 (Bonn), S. 202. Dem voraus ging der Entschluß der Zentralkomitees der drei Parteien v. 4. 12. 1977, eine entsprechende Vereinigung anzustreben, Novedades v. 5. 12. 1977, S. 1 u. 17. Der Versuch, zu Absprachen mit dem PPS zu kommen, war gescheitert, so der Generalsekretär des PCM Arnoldo Martínez Verdugo in einem Interview im ND v. 7. 8. 1976.

77 Diese Bewegung hatte sich 1950 als Absplitterung militanter Kommunisten vom PCM gebildet, Murillo S. a.a.O., S. 185, sie ist als asociación política nacional, der Vorstufe einer politischen Partei, registriert und hat damit die Möglichkeit, in Verbindung mit einer politischen Partei auf deren Liste an Wahlen teilzunehmen.

78 So stammten $62,63 \%$ der Stimmen des PCM aus dem D. F. und dem Bundesstaat México, Segovia (Anm. 25), S. 404. Hinzu kam, daß einige Stimmen dem PCM zufielen, um dessen endgültige Registrierung zu erreichen, ohne sich jedoch mit den politischen Zielen der Kommunisten nachhaltig zu identifizieren, vgl. die Bekenntnisse von Segovia und vom Sohn des Chefideologen Reyes Heroles in: Proceso Nr. 153 v. 8. 10. 1979, S. 6 f.

79 So konnte der PCM auch in Nayarit, der bisherigen Hochburg des PPS mehr als im Bundesdurchschnitt an Stimmen erhalten, Segovia (Anm. 25), S. 404. 
allein der PCM nach PRI und PAN als ernstzunehmende parlamentarische Kraft angesehen werden ${ }^{80}$. Angesichts der Zersplitterung der übrigen Linken wird allein dem PCM eine Chance gegeben, bei den Deputiertenwahlen 1982 die Linke zu repräsentieren ${ }^{81}{ }^{82}$. Einen guten Uberblick über die vor allem aus den Absplitterungen und Mitgliederausschlüssen des PCM entstandenen Gruppierungen geben die Arbeiten von Murillo S. und von Rodríguez $\mathrm{A}$. Zu erwähnen ist etwa der aus den: PCM ausgeschlossene Flügel reformistischer Provenienz, der sich als Unidad Izquierda Comunista - UIC - selbständig machte ${ }^{83}$ und sich neben dem bereits erwähnten MAUS nach dem Gesetz von 1977 registrieren ließ, indes auch nur als sog. asociación ${ }^{84}$.

Vornehmlich marxistisch-trotzkistischer Ideologie ${ }^{85}$ verschrieben haben sich die diversen "Arbeiter"-Parteien intellektueller Kreise, die für sich in Anspruch nehmen, "für die Arbeiter" und deren "wahren Interessen" zu sprechen. Im Gefolge der Studentenunruhen von 1968 gründeten Studenten den Partido Revolutionario de los Trabajadores PRT $-{ }^{86}$. Bereits vorher gab es kleinere trotzkistische Gruppierungen, so der 1959 als mexikanische Sektion der IV. Internationale gegründete Partido Obrero Revolucionario - POR -, von dem sich in den Auseinandersetzungen um den kubanischen Kommunismus 1960 die Liga Obrera Marxista - LOM - trennte, mit ganzen 20 Mitgliedern $^{87}$. Nach den Studentenunruhen von 1968 hielt sich eine kleine Gruppe mit Namen Grupo Comunista Internacionalista - GCI -, die schließlich im September 1976 mit der 1973 gegründeten Liga Socialista zum Partido Revolucionario de los Trabajadores fusionierte $e^{88}$.

Mit der Inkorporation weiterer trotzkistischer Gruppierungen im Jahre 1977 erhob die Partei den Anspruch, erstmals alleinige Vertreterin des Trotzkismus in Mexiko, wenn nicht gar in ganz Lateinamerika zu sein. Diese Partei ließ sich als asociación namens Movimiento por el Partido Revolucionario de los Trabajadores registrieren ${ }^{89}$.

80 So Segovia (Anm. 25), S. 404/5; Latin America - weekly report v. 20. 3. 1981, S. 6/7.

81 Hierin sieht Segovia, Excelsior v. 20. 2. 1981, eine Chance für das mexikanische Wahlsystem, wenn sich lediglich eine Rechte, eine Linke und das Zentrum dem Wähler stellen.

82 Lokale Erfolge bei Gemeindewahlen, deren Respektierung durch den PRI auf dieser Ebene als Triumph der Demokratie gef eiert wird, geben sicherlich künftige Stützpunkte ab: In Juchitán/Oaxaca gewann eine Koalition von PCM und einer Coalición Obrera, Campesina y Estudiantil del Istmo (d. h. von Tehuantepec) vor der des PRI mit dem PPS, uno mas uno v. 4. 3. 1981.

83 Murillo S. a.a.O., S. 182 f.

84 Javier López Moreno, La Reforma Política en México, México 1979, S. 257, 262 ff. Als weitere asocación will sich 1981 die seit 17 Jahren bestehende Asociación Nacional de Egresados de Excuelas Superiores para Hijos de Trabajadores A. C. als Asociación Política Cardenista registrieren lassen, Excelsior v. 20. 5. 1981, S. $18 \mathrm{~A}$.

85 Nicht zu vergessen ist, daß Trotzki in Mexiko auf Einladung des Gewerkschaftsführers Lombardo Toledano Exil gef unden hatte.

86 Murillo S. a.a.O., S. 184 f. Jetzt als Partei zugelassen, uno mas uno v. 12.6. 1981.

87 Rodríguez A. a.a.O., S. 205/6.

88 Rodríguez A. a.a.O., S. 206-209. Die von beiden Gründungsorganisationen herausgegebenen Zeitschriften schlossen sich zusammen zur "Bandera Sozialista", seither offizielles Publikationsorgan der Partei.

89 López M. (Anm. 84), S. 271 ff. In Baja California Sur ist sie als Landespartei am 4. 7. 1980 registriert worden, Gaceta informativa (Legislación y jurisprudencia) Nr. 31 (Sept.-Dez. 1980), S. 670. 
Mit dem PRT vom Namen her nicht zu verwechseln ist der Partido Socialista de los Trabajadores - PST - und dieser wiederum nicht mit dem Partido Mexicano de los Trabajadores - PMT -.

Beider Ursprung ist in dem unter maßgeblicher Beteiligung von dem Schriftsteller Carlos Fuentes 1971 gegründeten Comité Nacional de Auscultación y Coordinación CNAC - zu sehen ${ }^{90}$. Nachdem bereits andere Gruppen das CNAC verlassen hatten, spaltete sich 1973 eine weitere Gruppe unter Rafael Aguilar Talamantes ab und bildete das Comité Organizador y de Consulta - COR -, das 1975 den PST gründete ${ }^{91}$.

Diese Partei, die eine gewisse Anhängerschaft im ländlichen Bereich hat ${ }^{92}$, steht nicht in grundsätzlicher Opposition zur Regierung, sondern strebt sogar eine "Alianza Popular Revolucionaria" mit ihr, genauer mit ihrem linken Flügel $a^{93}$. Der PRI zeigte sich durch direkte und indirekte Hilfen erkenntlich. Die Konkurrenz zur bisherigen Rolle des weitgehend diskreditierten PPS wird deutlich. Der PST ist nun deswegen für das neue mexikanische Parteiensystem interessant, weil mit ihm die Brücke geschlagen ist zu den terroristischen Gruppierungen, denen damit der Weg zurück in die Legalität eröffnet wird ${ }^{94}$. Der PST setzt sich militant für Landbesitz der Eingeborenen ein - so im unruhigen Huastekengebiet $-{ }^{95}$ und mit seiner Frauenorganisation ${ }^{96}$ für die völlige Legalisierung der Abtreibung ${ }^{97}$.

Bei den Wahlen 1979 erreichte er 2,26\% der (Zweit-)Stimmen ${ }^{98}$ und damit 10 Listenmandate.

Der Partido Mexicano de los Trabajadores wurde im September 1974 von Heberto Castillo gegründet ${ }^{99}$, vorbereitet von dem erwähnten Rumpf-Komitee - CNAC -, Ende 1972 umbenannt in Comité Nacional de Auscultación y Organización - CNAO - ${ }^{100}$. Die Partei $^{101}$ verschreibt sich dem Fortschritt der Demokratie nicht bloß als Ziel, sondern als Notwendigkeit für die Mobilisation und strukturelle Veränderung der Massen ${ }^{102}$. Rückhalt sucht die Partei in den zum Gewerkschaftsbund CTM in Opposition stehenden Eisenbahner-, Elektriker-, Transportarbeiter- und Minenarbeitergewerkschaften ${ }^{103}$. Für

90 Rodríguez A. a.a.O., S. $179 \mathrm{ff}$.

91 Lehr a.a.O., S. $170 \mathrm{ff}$.

92 Proceso Nr. 186 v. 26. 5. 1980 , S. 35.

93 Lehr a.a.O., S. 172.

94 Nach Bekundung seines Vorsitzenden Rafael Aguilar Talamantes kandidierten für den PST bei den Wahlen 197913 ehemalige Guerillakämpfer, vgl. Entwicklungspolitik kommunistischer Länder II/1978 (Bonn), S. 202. In den letzten drei Jahren sollen etwa 40 Angehörige des PST in den südwestlichen Bundesstaaten erschossen worden sein, Excelsior v. 20. 5. 1981, S. 18 A.

$95 \mathrm{Er}$ wird daher als Urheber der illegalen Landbesetzungen angeschuldigt, uno mas uno v. 5. 3. 1980, S. 7. Hierzu bekennt er sich im Bundesstaat Veracruz, vgl. Excelsior v. 20. 5. 1981, S. 28 A.

96 Federación Nacional de Mujeres insurgentes.

97 El Día v. 10. 3. 1980, S. 3.

98 Und ist demnach endgültig registriert, D. O. v. 6. 9. 1979.

99 Rafael Segovia, in: L'ordinaire du Mexicaniste Nr. 21 (Perpignan März 1977), S. 23 ff. (31).

100 Zur Vorgeschichte Rodríguez A. a.a.O., S. 179 ff.

101 Für die Wahlen 1982 jedoch nicht registriert, uno mas uno v. 12.6. 1981.

102 Rodríguez A. a.a.O., S. 183.

103 Rodríguez A. a.a.O., S. 184. Nach eigenen Angaben soll der PMT 13000 Mitglieder haben, NZZ v. 6. 1. 1978. 
das innenpolitische Tagesgeschehen sucht sie die Zusammenarbeit mit anderen Linksparteien, so etwa auch mit dem PPM. Plakative Forderungen ${ }^{104}$ sind die Erhöhung der Mindestlöhne und die Einführung der 35-Stunden-Woche. Eine publizistische Plattform eröffnet sich dem PMT in der im März 1980 gegründeten Halbmonatszeitschrift Crítica Política $^{105}$.

Als durchaus rechtsextremistische Partei ist der Partido Demócrata Mexicano - PDM zu sehen, dessen Anhängerschaft sich aus dem sinarquistischen Umfeld rekrutiert. Der in den dreißiger Jahren entstandene Sinarquismus (sino anarquismo - ohne Anarchie) verstand sich als ländlich-kleinbäuerliche Bewegung gegen die als kommunistisch/anarchistisch angesehene Reformpolitik von Staatspräsident Cárdenas. Personelle Uberschneidungen mit der Cristero-Bewegung waren zwangsläufig ${ }^{106}$, bezeichnend daher auch, daß der regionale Schwerpunkt der Sinarquisten in den zentralen westlichen Bundesstaaten liegt ${ }^{107}$. Die verschiedenen lokalen Gruppierungen ${ }^{108}$ schlossen sich of fiziell ${ }^{109}$ am 23. Mai 1937 in León/Gto. zur Unión Nacional Sinarquista - UNS - zusammen. Da diese sich selbst vornehmlich als geistige Bewegung versteht ${ }^{110}$, mußte für die Beteiligung im tagespolitischen Kampf, insbesondere für die Wahlen, eine politische Partei von der UNS gegründet werden. Diese war die Fuerza Popular, die an den Wahlen von 1946 teilnehmen konnte und ein Mandat in Guanajuato errang. Die Regierungspartei wurde jedoch auch mit dieser Bewegung mit einer typischen Doppelstrategie fertig: Die Regierungspartei selbst rückte deutlich nach rechts unter dem General Avila Camacho. Auf der anderen Seite wurde mit der Ley Electoral von 1951 der Nachweis von wenigstens 75000 Anhängern (statt bisher 30 000) verlangt, den die Fuerza Popular sicher hätte erreichen können, jedoch in zwei Dritteln aller Bundesstaaten: Angesichts der lokalen Bedeutung der Sinarquistenbewegung war dies Erfordernis schwer zu erreichen, die Fuerza Popular verzichtete auf eine Registrierung.

Schließlich wurde als politische Partei des Sinarquismus im Gefolge der von Staatspräsident Echeverría (1970-1976) verheißenen apertura democrática im Februar/März 1972 der PDM gegründet, der nach dreijähriger Aufbauarbeit in den Bundesstaaten am 29. Juni 1975 den Registrierungsantrag stellte ${ }^{111}$, dem dann erst 1978 stattgegeben wurde. Seine sinarquistische Ausrichtung konnte der PDM dokumentieren, indem er, als der

104 Abgelesen von entsprechenden Wandparolen in México Ciudad.

105 Vgl. Nr. 1 v. 15.-30. 3. 1980, S. 15-17, in der der Präsident des Nationalkomitees des PMT, Heberto Castillo, ausgiebig zu Wort kommt.

106 Die katholische Kirche indes hatte sich vom Sinarquismus distanziert, Willliam P. Tucker, The Mexican Government Today, 2. Aufl. Minneapolis 1958, S. 35.

107 Aguascalientes, Guanajuato, Jalisco, Michoacan, vgl. die Karte bei Anne Marie de la Vega-Leinert, in: Comercio Exterior 1976, S. 1076 ff. (1083).

108 Unter ihnen das Centro Anti-Comunista des Deutschen Hellmut Oscar Schreiter, Moreno a.a.O., S. 150/1.

109 Gemeint, sie traten offen als der "sichtbare Teil" der geheimen Organisation "Las Legiones" auf, de la Vega-Leinert (Anm. 107), S. 1077.

110 Vgl. das bei Moreno a.a.O., S. 157/8, abgedruckte Manifiesto del Comité Organizador Sinarquista al Pueblo Mexicano v. 12. 6. 1937: "El Sinarquismo es un modo de ser y de vivir; . . Es una actividad espiritual ...".

111 Rodríguez A. a.a.O., S. 170. 
PRI als Gouverneurskandidaten für Michoacán Cuauhtémoc Cárdenas, den Sohn des vormaligen Staatspräsidenten, aufstellte ${ }^{112}$, warnend auf den noch lebenden Cardenismo hinwies ${ }^{113}$. Als Kandidat des PDM wurde mit Alfonso López Camacho ein alter militanter Sinarquist entgegengestellt ${ }^{114}$. Der PDM im allseitigen Kampf gegen die Linksparteien wie auch gegen die ernst zu nehmende Konkurrenz des PAN ${ }^{115}$ gelingt es immer wieder, von sich sprechen zu machen: So stellte er als Kandidat für das Gouverneursamt in Zacatecas eine Frau, die Bundesdeputierte Adelaide Márquez, auf ${ }^{116}$. Bedroht von internen Flügelkämpfen ${ }^{117}$ und von neuen rechtsextremistischen Gruppierungen ${ }^{118}$, gelang es dem PDM bei den Wahlen vom 1. Juli 1979, mit 293540 (Zweit-)Stimmen 2,13\% der Stimmen zu erringen ${ }^{119}$.

IV.

Dieser angesichts der vorgegebenen Thematik begrenzte Ausschnitt aus dem politischen Geschehen Mexikos ${ }^{119 a}$ darf nicht übersehen lassen, daß es gegenüber der nach wie vor erdrückenden Úbermacht der Regierungspartei nur eine wirkliche gleichgewichtige Alternative gibt, nämlich die Partei der Nichtwähler. Diese von dem System in durchaus zutreffender Beurteilung als "partido negativo" gekennzeichnete Wählerenthaltung ist nach der Reform von 1977 keineswegs kleiner, sondern sogar noch größer geworden ${ }^{120}$. 1979 blieben über $50 \%$ der Wähler zu Hause, angesichts der Tatsache, daß sich doch nichts entscheidend ändern lassen würde, eine politisch konsequente Haltung. Erst die Mobilisation anläßlich der Präsidentschaftswahl von $1982^{121}$ mit zu erwartender hoher Wahlbeteiligung wird dieses Debakel von 1979 überdecken können.

112 Um des symbolträchtigen Namens prompt vom PCM, PST und PPS unterstützt, Excelsior v. 3. 3. 1980, S. 27 A.

113 Excelsior v. 3. 3. 1980 , S. 4 A und 27 A.

114 Diario de México v. 20. 3. 1980, S. 6.

115 So rügte der Sprecher des PDM, Jesús Zamora Flores, den PAN und den PCM als nur destruktiv, da diese Parteien keine konstruktiven Lösungsvorschläge unterbreiten könnten, El Heraldo v. 14. 3. 1980, S. 2 A.

116 Diario de México v. 20. 3. 1980, S. 6.

117 Rafael Segovia (Anm. 99), S. 34. El Día v. 26. 5. 1981, S. 3.

118 So ein antikommunistischer Partido de Reconstrucción Nacional, publizistisch unterstützt durch die Zeitschrift Impacto, vgl. Nr. 1569 v. 26. 3. 1980, S. 9 f. Zum Standort dieser Zeitschrift vgl. weiter Gerhard Scheffler, in: Uberseeische Verfassungsvergleichung I (Hamburg 1980), S. 35 ff. (51/2).

119 Demgemäß wurde er definitiv als politische Partei registriert, D. O. v. 6. 9. 1979.

119a Von 16 neuen Organisationen wurden für 1982 nur 2 zugelassen, neben dem PRT (Anm. 86) der Partido Social Demócrata (PSD), uno mas uno v. 12.6. 1981.

120 Von of fizieller Seite wird die Wahlenthaltung allen Parteien als "partidos despolitizadores" angelastet, Novedades v. 6. 5. 1981, S. 4.

121 Kandidat des PR I ist Miguel de la Madrid, Die Welt v. 29. 9. 1981, S. 6, d. h. der künftige Präsident Mexikos $1982-1988$. 
nalism, uniting the nation behind the king and covering other problems. The opposed Socialist Union of the People's Forces (USFP) is suffering considerable repressions. In the Democratic People's Republic of Algeria the institutional system is based on the principle of one single party (Art. 94) of the Constitution of 1976. This option resulted from the hard war of liberation carried on by the National Front of Liberation (FLN) which now, according to Art. 95 of the Constitution, was declared the unitary party and the leader of the socialist orientation. The Party and the State are mixed up; but the State is still the determinant factor. The regime is consolidated and supported by the broad majority of the people. A certain peripheral opposition could not gain any real importance.

The liberal-socialist Republic of Tunesia is between the two above mentioned systems. Because of the majority-suffrage and certain administrative practises the Socialist Destour (Constitution) Party (PSD) of President Bourguiba has become a de facto unitary party. The Party is based on the principle of the insoluble unity of the various social classes and considers itself as the representative of the whole tunesian people. But in the last few years the monopoly of the Party was made more and more dubious and a political opening was claimed. Under this pressure, in April 1981, Bourguiba accepted on principle the system of pluralism and - however under certain reservations - the recognition of other parties. Since then various parties have already asked for admission. It is remarkable that the first party to be admitted besides the PSD was the Communist Party of Tunesia (PCT) because it had already existed before and was only "suspended ". Under the new regime of the political opening anticipated elections for the Parliament shall take place already on the 1st of November, 1981.

\section{The Minor Political Parties of Mexico by Gerhard Scheffler}

In relationship to the ruling ubiquitous PRI - Institutional Revolutionary Party - all of the other parties in Mexico are substantielly of no relevant importance. There are three groups of these parties: The principal legal opposition party, the PAN - National Action Party -; the other legally registered parties and nowadays the new or recently registered parties.

The 1939 founded conservative Catholic PAN has had no real chance to win elections in the former majority system, but it contributes to the very facade of democracy which the PRI wished to perpetuate.

The PPS - Popular Socialist Party -, founded by the Marxist intellectual Lombardo Toledano 1947 is no better than a satellite party of the PRI on the left of the political spectrum. Like its so-called leftist counterpart the slightly right-of-center PARM - Authentic Party of the Mexican Revolution - is an appendage of the PRI. 
The constitutional and electoral reform of 1977 establishing a combined system of majority and proportional representation allowed the registration of the Communist Party PCM - and the foundation of some new "Workers Parties" on the left and of the PDM Mexican Democratic Party - on the right.

The electoral reform produces no more political mobilisation but an abstention about 50 percent in the elections of 1979 , theref ore the real counterpart of ruling PRI is the party of non-voters. A change to more voting we will have in 1982 for the presidential elections. Candidate of PRI and therefore very presumable next Mexican president 1982-1988 is Miguel de la Madrid. 